\title{
SPACINGS BETWEEN INTEGERS HAVING TYPICALLY MANY PRIME FACTORS
}

\author{
RIZWANUR KHAN
}

\begin{abstract}
We show that the sequence of integers which have nearly the typical number of distinct prime factors forms a Poisson process. More precisely, for $\delta$ arbitrarily small and positive, the nearest neighbor spacings between integers $n$ with $\left|\omega(n)-\log _{2} n\right| \leq\left(\log _{2} n\right)^{\delta}$ obey the Poisson distribution law.
\end{abstract}

\section{INTRODUCTION}

Consider $n$ random variables independently and uniformly taking real values in the interval $[0, n]$. Let $Y_{1}<\ldots<Y_{n}$ denote the order statistics obtained by arranging these random variables in increasing order. Setting $Y_{0}=0$ and $Y_{n+1}=n$, let $D_{i}=Y_{i+1}-Y_{i}$ for $0 \leq i \leq n$ denote the nearest neighbor spacings of the order statistics. Thus $D_{1}+\ldots+D_{n}=n$ and by symmetry it follows that for $0<\lambda<n$ a real number,

$$
\operatorname{Prob}\left(D_{i}>\lambda\right)=\operatorname{Prob}\left(D_{1}>\lambda\right)=\left(\frac{n-\lambda}{n}\right)^{n} .
$$

Thus $\operatorname{Prob}\left(D_{i}>\lambda\right) \sim e^{-\lambda}$ as $n \rightarrow \infty$. This is the exponential or Poisson distribution.

We are interested in the spacing distributions of arithmetic sequences. An example of such a sequence is the sequence of prime numbers less than $x$, which form a sparse subset of the integers of density $1 / \log x$ by the prime number theorem. This is similar to $Y_{1}, \ldots, Y_{n}$ being sparse in the interval $[0, n]$. If $p_{i}$ denotes the $i$-th prime less than $x$, we rescale to consider instead the sequence $\widetilde{p}_{i}=p_{i} / \log x$ of 'normalized' primes so that that the average spacing between consecutive normalized primes is 1 as $x \rightarrow \infty$. This matches the expected value of $D_{i}$ above as $n \rightarrow \infty$. Gallagher [4] showed that assuming the validity of the Hardy-Littlewood prime $k$-tuple conjectures, we have for $\lambda>0$ real that

$$
\frac{1}{x} \#\left\{i \leq x: \widetilde{p}_{i+1}-\widetilde{p}_{i}>\lambda\right\} \sim e^{-\lambda}
$$

as $x \rightarrow \infty$. Thus conditionally we see that the spacings between primes obey the Poisson distribution law, as in the prototypical situation of randomly dispersed objects mentioned at the start. More recently Kurlberg and Rudnick [9] showed that the spacings between quadratic residues modulo $q$, as the number of distinct prime divisors of $q$ tends to infinity, follow the Poisson distribution. There are many other interesting arithmetic sequences that are conjectured to be Poisson processes, but only few examples exist with proof. For example, it is an open problem to show that the spacings between the fractional parts of $n^{2} \sqrt{2}$ for $n \leq x$, as $x \rightarrow \infty$ are Poisson distributed (see [11). The reader may find a few more examples of such work listed in the references section (see $[1,6,77$ ). Of course there are important 
arithmetic sequences which are not expected to behave like randomly dispersed elements in this sense, such as the non-trivial zeros of the Riemann Zeta function. In this paper we are interested in the spacings between integers with not only one prime factor as in Gallagher's work, but with the typical number of distinct prime factors. We first explain what is meant by 'typical'.

Let $\omega(n)$ denote the number of distinct prime factors of $n$. It is easy to see that integers $n \leq x$ have $\log \log x$ distinct prime factors on average:

$$
\frac{1}{x} \sum_{n \leq x} \omega(n)=\frac{1}{x} \sum_{n \leq x} \sum_{p \mid n} 1=\frac{1}{x} \sum_{p \leq x} \sum_{\substack{n \leq x \\ p \mid n}} 1=\frac{1}{x} \sum_{p \leq x}\left\lfloor\frac{x}{p}\right\rfloor=\log _{2} x+O(1),
$$

where we write $\log _{2} x$ for $\log \log x$, and similarly for $\log _{j} x$. Also, throughout this paper $p$ and $q$ will be used to denote primes. The variance can be shown to be

$$
\frac{1}{x} \sum_{n \leq x}\left(\omega(n)-\log _{2} x\right)^{2} \sim \log _{2} x .
$$

Note that (1.2) and (1.3) imply that $\omega(n) \sim \log _{2} x$ for all most all $n \leq x$. Erdös and Kac 2 further showed that $\omega(n)$ is normally distributed with mean $\log _{2} x$ and standard deviation $\sqrt{\log _{2} x}$. Rényi and Turán [10] proved this result with a sharp error term. The following theorem can also be found in Tenenbaum's book [14].

Theorem 1.1. Given a real number $C>0$ we have for $0<c<C$ that the number of integers $n \leq x$ for which $-c<\frac{\omega(n)-\log _{2} x}{\sqrt{\log _{2} x}}<c$ is

$$
x \frac{1}{\sqrt{2 \pi}} \int_{-c}^{c} \exp \left(-u^{2} / 2\right) d u+O_{C}\left(x / \sqrt{\log _{2} x}\right) .
$$

We [8] proved a slightly weaker version of the Theorem 1.1 by methods similar to those in this paper.

We conjecture that the spacings between integers $n \leq x$ with $\left|\omega(n)-\log _{2} x\right| \leq$ $\sqrt{\frac{\pi}{2}}$ (that is, integers with more or less exactly $\log _{2} x$ distinct prime factors) obey the Poisson distribution law but we are unable to prove it. Instead we look at an easier question. For any fixed $0<\delta<1 / 2$, let us say an integer less than $x^{2}$ is ' $\delta$-normal' if

$$
\left|\omega(n)-\log _{2} x\right| \leq \sqrt{\frac{\pi}{2}}\left(\log _{2} x\right)^{\delta} .
$$

We study the sequence of $\delta$-normal numbers. These are integers having nearly the expected number of prime factors, as $\left(\log _{2} x\right)^{\delta}$ is smaller than the standard deviation $\sqrt{\log _{2} x}$ of $\omega(n)$. Denote the sequence of $\delta$-normal numbers in increasing order by $N_{1}, N_{2}, N_{3} \ldots$. Up to $x$, there are $x\left(\log _{2} x\right)^{-1 / 2+\delta}$ such integers by Theorem 1.1] since an integer is $\delta$-normal if and only if $\left|\frac{\omega(n)-\log _{2} x}{\sqrt{\log _{2} x}}\right| \leq \sqrt{\frac{\pi}{2}}\left(\log _{2} x\right)^{-1 / 2+\delta}$. Thus we should rescale these integers by setting $\widetilde{N}_{i}=N_{i}\left(\log _{2} x\right)^{-1 / 2+\delta}$. Our main theorem is

Theorem 1.2. For any fixed real number $\lambda>0$ we have

$$
\frac{1}{x} \#\left\{i \leq x: \widetilde{N}_{i+1}-\tilde{N}_{i}>\lambda\right\} \sim e^{-\lambda} .
$$

Throughout this paper, all implicit constants may depend on $\delta$ and $\lambda$. 


\section{INDEPENDENCE BETWEEN ADDITIVE ShIFTS OF THE $\omega(n)$ FUNCTION}

In this section we show how Theorem 1.2 can be reduced to studying correlations between the additive shifts of the function $\omega(n)$. We will show for example that $\omega(n)-\log _{2} x, \omega(n+1)-\log _{2} x$, and $\omega(n+2)-\log _{2} x$ behave independently. Define $\mathcal{N}(x)$ to be the number of $\delta$-normal integers less than $x$. The left hand side of Theorem 1.2 is asymptotic to

$$
\begin{aligned}
& \frac{1}{x} \#\left\{i \leq x: N_{i+1}-N_{i}>\lambda\left(\log _{2} x\right)^{1 / 2-\delta}\right\} \\
& \sim \frac{1}{x} \#\left\{N \leq x\left(\log _{2} x\right)^{1 / 2-\delta}: \mathcal{N}\left(N+\lambda\left(\log _{2} x\right)^{1 / 2-\delta}\right)-\mathcal{N}(N)=0\right\},
\end{aligned}
$$

where $N$ denotes a $\delta$-normal number. Define $\mathcal{N}_{b_{1}, \ldots, b_{r}}(x)$ to be the number of integers $n \leq x$ for which $n+b_{i}$ is $\delta$-normal for all $1 \leq i \leq r$ and let $\sigma(m, r)$ denote the number of maps from the set $\{1, \ldots, m\}$ onto $\{1, \ldots, r\}$. We have the $m$-th moment of $\mathcal{N}\left(N+\lambda\left(\log _{2} x\right)^{1 / 2-\delta}\right)-\mathcal{N}(N)$ :

$$
\begin{aligned}
& \frac{1}{x} \sum_{N \leq x\left(\log _{2} x\right)^{1 / 2-\delta}}\left(\mathcal{N}\left(N+\lambda\left(\log _{2} x\right)^{1 / 2-\delta}\right)-\mathcal{N}(N)\right)^{m} \\
& =\frac{1}{x} \sum_{r=1}^{m} \sigma(m, r) \sum_{1 \leq b_{1}<\ldots<b_{r} \leq \lambda\left(\log _{2} x\right)^{1 / 2-\delta}} \mathcal{N}_{0, b_{1}, \ldots, b_{r}}\left(x\left(\log _{2} x\right)^{1 / 2-\delta}\right) .
\end{aligned}
$$

We will prove

Theorem 2.1. For a fixed integer $r$ and any integers $0 \leq b_{1}<\ldots<b_{r} \leq$ $\lambda\left(\log _{2} x\right)^{1 / 2-\delta}$, we have

$$
\frac{1}{x} \mathcal{N}_{b_{1}, \ldots, b_{r}}(x) \sim\left(\log _{2} x\right)^{(-1 / 2+\delta) r} .
$$

Throughout this paper all implicit constants may depend $r$. Since a randomly chosen integer less than $x$ is $\delta$-normal with probability $\left(\log _{2} x\right)^{-1 / 2+\delta}$, the theorem above says that $n+b_{1}, \ldots, n+b_{r}$ are independently likely to be $\delta$-normal. Theorem 2.1 implies that for fixed $m$ we have that (2.2) is asymptotic to

$$
\sim \sum_{r=1}^{m} \sigma(m, r) \frac{\lambda^{r}}{r !}=\sum_{j=0}^{\infty} j^{m} \frac{e^{-\lambda} \lambda^{j}}{j !}
$$

which is the $m$-th moment of the Poisson distribution (the identity above is known as Dobinski's formula). The Poisson distribution can be recovered from these moments. Let us sketch this; we have that (2.1) is

$$
\begin{aligned}
& \frac{1}{x} \#\left\{N \leq x\left(\log _{2} x\right)^{1 / 2-\delta}: \mathcal{N}\left(N+\lambda\left(\log _{2} x\right)^{1 / 2-\delta}\right)-\mathcal{N}(N)=0\right\} \\
& \sim 1-\frac{1}{x} \sum_{j=1}^{\infty} \sum_{\substack{N \leq x\left(\log _{2} x\right)^{1 / 2-\delta} \\
\mathcal{N}\left(N+\lambda\left(\log _{2} x\right)^{1 / 2-\delta}\right)-\mathcal{N}(N)=j}}^{1} \sum_{\mathcal{N}\left(N+\lambda\left(\log _{2} x\right)^{1 / 2-\delta}\right)-\mathcal{N}(N)=j} 1 .
\end{aligned}
$$


Now

$$
\frac{1}{x} \sum_{j=1}^{\infty} j^{m} \sum_{\substack{N \leq x\left(\log _{2} x\right)^{1 / 2-\delta} \\ \mathcal{N}\left(N+\lambda\left(\log _{2} x\right)^{1 / 2-\delta}\right)-\mathcal{N}(N)=j}} 1
$$

is the $m$-th moment of $\mathcal{N}\left(N+\lambda\left(\log _{2} x\right)^{1 / 2-\delta}\right)-\mathcal{N}(N)$. By (2.3) we get (an explicit dependence on $m$ of the error term is not needed) that (2.4) is asymptotic to

$$
\sim 1-\sum_{j=1}^{\infty} \sum_{m=0}^{\infty} \frac{(2 \pi \mathbf{i})^{m}}{m !} j^{m} \frac{e^{-\lambda} \lambda^{j}}{j !}=e^{-\lambda}
$$

Thus Theorem 1.2 follows from Theorem 2.1. Next we discuss the demonstration of Theorem 2.1

The characteristic function of a random variable with a normal distribution is $\exp \left(-T^{2} / 2\right)$. We show the independence of $\frac{\omega\left(n+b_{i}\right)-\log _{2} x}{\sqrt{\log _{2} x}}$ for $1 \leq i \leq r$ by showing that their joint characteristic function equals essentially $\prod_{i=1}^{r} \exp \left(-T_{i}^{2} / 2\right)$. Actually it is more convenient to work with $\omega(n ; y, z)$ in place of $\omega(n)$, where we set

$$
y=y(x)=(\log x)^{3 r}
$$

and

$$
z=z(x)=x^{\left(\left(\log _{2} x\right)^{-3 r}\right)}
$$

and define

$$
\omega(n ; y, z)=\sum_{\substack{p \mid n \\ y<p<z}} 1 .
$$

Accordingly we work with $\omega(n ; y, z)-\sum_{y<p<z} \frac{1}{p}$ in place of $\omega(n)-\log _{2} x$. We will soon see that there is not much loss in disregarding the primes less than $y$ or greater than $z$. In an imprecise sense, the reason for this is that on average integers have few small prime factors and few large prime factors. In the next section we will prove the following theorem.

Theorem 2.2. Let $t_{i}=T_{i}\left(\sum_{y<p<z} \frac{1}{p}\right)^{-1 / 2}$ be real. For $\left|T_{i}\right| \leq \frac{1}{1000}\left(\sum_{y<p<z} \frac{1}{p}\right)^{1 / 2}$, we have

$$
\begin{gathered}
\frac{1}{x} \sum_{n \leq x} \prod_{i=1}^{r} \exp \left(\mathbf{i} T_{i} \frac{\omega\left(n+b_{i} ; y, z\right)-\sum_{y<p<z} \frac{1}{p}}{\sqrt{\sum_{y<p<z} \frac{1}{p}}}\right)=\prod_{i=1}^{r} \exp \left(\left(e^{\mathbf{i} t_{i}}-1-\mathbf{i} t_{i}\right) \sum_{y<p<z} \frac{1}{p}\right) \\
+O(1 / \log x) .
\end{gathered}
$$

Observe that for $T_{i} \leq\left(\log _{2} x\right)^{\epsilon}$ for small enough $\epsilon>0$ we have

$$
\begin{aligned}
\exp \left(\left(e^{\mathbf{i} t_{i}}-1-\mathbf{i} t_{i}\right) \sum_{y<p<z} \frac{1}{p}\right) & =\exp \left(\left(\frac{-t_{i}^{2}}{2}+O\left(t_{i}^{3}\right)\right) \sum_{y<p<z} \frac{1}{p}\right) \\
& =\exp \left(\frac{-T_{i}^{2}}{2}\right)\left(1+O\left(\frac{T_{i}^{3}}{\sqrt{\log _{2} x}}\right)\right),
\end{aligned}
$$


and for $\left(\log _{2} x\right)^{\epsilon}<T_{i} \leq\left(\log _{2} x\right)^{1 / 2-\epsilon}$ we have

$$
\exp \left(\left(e^{\mathrm{i} t_{i}}-1-\mathbf{i} t_{i}\right) \sum_{y<p<z} \frac{1}{p}\right) \ll \exp \left(-T_{i}^{2} / 4\right),
$$

where the implied constants depend on $\epsilon$.

To see how Theorem 2.2 implies Theorem 2.1 we will use the following lemmas.

Lemma 2.3. Let $\psi(x)$ be a real function differentiable $\lfloor 4 r / \delta\rfloor$ times and satisfying

$$
\begin{aligned}
& 0 \leq \psi(x) \leq 1 \text { for } x \in \mathbb{R}, \\
& \psi(x)=0 \text { for }|x| \geq 2\left(\log _{2} x\right)^{-1 / 2+\delta} \\
& \int_{-\infty}^{\infty} \psi(x) d x \sim \sqrt{2 \pi}\left(\log _{2} x\right)^{-1 / 2+\delta}, \\
& \left|\psi^{(j)}(x)\right| \ll\left(\log _{2} x\right)^{j(1-\delta) / 2} \text { for any positive integer } j \leq\lfloor 4 r / \delta\rfloor .
\end{aligned}
$$

We have

$$
\frac{1}{x} \sum_{n \leq x} \prod_{i=1}^{r} \psi\left(\frac{\omega\left(n+b_{i} ; y, z\right)-\sum_{y<p<z} \frac{1}{p}}{\sqrt{\sum_{y<p<z} \frac{1}{p}}}\right) \sim\left(\log _{2} x\right)^{(-1 / 2+\delta) r} .
$$

Proof. Let

$$
\hat{\psi}(T)=\int_{-\infty}^{\infty} \psi(u) e^{-\mathbf{i} u T} d u
$$

denote the Fourier transform of $\psi$. By Fourier inversion we have

$$
\begin{aligned}
& \frac{1}{x} \sum_{n \leq x} \prod_{i=1}^{r} \psi\left(\frac{\omega\left(n+b_{i} ; y, z\right)-\sum_{y<p<z} \frac{1}{p}}{\sqrt{\sum_{y<p<z} \frac{1}{p}}}\right) \\
& =\frac{1}{x} \sum_{n \leq x} \prod_{i=1}^{r} \frac{1}{2 \pi} \int_{-\infty}^{\infty} \hat{\psi}\left(T_{i}\right) \exp \left(\mathbf{i} T_{i} \frac{\omega\left(n+b_{i} ; y, z\right)-\sum_{y<p<z} \frac{1}{p}}{\sqrt{\sum_{y<p<z} \frac{1}{p}}}\right) d T_{i} .
\end{aligned}
$$

We have that $\left|\hat{\psi}\left(T_{i}\right)\right| \ll\left(\log _{2} x\right)^{j(1-\delta) / 2}\left|T_{i}\right|^{-j}$, by integrating by parts $j$ times and using (2.8). Thus (2.9) equals

$$
\begin{aligned}
& \frac{1}{x} \sum_{n \leq x} \prod_{i=1}^{r} \frac{1}{2 \pi} \int_{-\left(\log _{2} x\right)^{1 / 2-\delta / 4}}^{\left(\log _{2} x\right)^{1 / 2-\delta / 4}} \hat{\psi}\left(T_{i}\right) \exp \left(\mathbf{i} T_{i} \frac{\omega\left(n+b_{i} ; y, z\right)-\sum_{y<p<z} \frac{1}{p}}{\sqrt{\sum_{y<p<z} \frac{1}{p}}}\right) d T_{i} \\
& \quad+O\left(\left(\log _{2} x\right)^{-r}\right) .
\end{aligned}
$$

Now by Theorem 2.2 and observations (2.6) and (2.7), the main term above equals

$$
\begin{aligned}
& \prod_{i=1}^{r}\left(\frac{1}{2 \pi} \int_{-\left(\log _{2} x\right)^{\epsilon}}^{\left(\log _{2} x\right)^{\epsilon}} \hat{\psi}\left(T_{i}\right) \exp \left(\frac{-T_{i}^{2}}{2}\right) d T_{i}+O\left(\frac{1}{\sqrt{\log _{2} x}}\right)\right) \\
& =\left(\frac{1}{2 \pi} \int_{-\infty}^{\infty} \hat{\psi}(T) \exp \left(\frac{-T^{2}}{2}\right) d T+O\left(\frac{1}{\sqrt{\log _{2} x}}\right)\right)^{r} .
\end{aligned}
$$


Recall that the Fourier transform of $\frac{1}{\sqrt{2 \pi}} \exp \left(\frac{-u^{2}}{2}\right)$ is $\exp \left(\frac{-T^{2}}{2}\right)$. By the Plancherel formula, (2.11) equals

$$
\left(\frac{1}{\sqrt{2 \pi}} \int_{-\infty}^{\infty} \psi(u) \exp \left(\frac{-u^{2}}{2}\right) d u+O\left(\frac{1}{\sqrt{\log _{2} x}}\right)\right)^{r} \sim\left(\log _{2} x\right)^{(-1 / 2+\delta) r} .
$$

To prove Theorem 2.1 we need to show

$$
\frac{1}{x} \sum_{n \leq x} \prod_{i=1}^{r} \psi\left(\frac{\omega\left(n+b_{i}\right)-\log _{2} x}{\sqrt{\log _{2} x}}\right) \sim\left(\log _{2} x\right)^{(-1 / 2+\delta) r},
$$

where $\psi$ is a suitable smooth function approximating the characteristic function of the interval $\left[-\sqrt{\frac{\pi}{2}}\left(\log _{2} x\right)^{-1 / 2+\delta}, \sqrt{\frac{\pi}{2}}\left(\log _{2} x\right)^{-1 / 2+\delta}\right]$. This is accomplished by Lemma 2.3. provided that we can show that we may neglect prime factors smaller than $y$ or larger than $z$ without significant loss. This is the purpose of the next lemma.

Lemma 2.4. Except for $O\left(x\left(\log _{2} x\right)^{-r}\right)$ integers less than $x$ we have

$$
\left|\frac{\omega(n)-\log _{2} x}{\sqrt{\log _{2} x}}-\frac{\omega(n ; y, z)-\sum_{y<p<z} \frac{1}{p}}{\sqrt{\sum_{y<p<z} \frac{1}{p}}}\right| \ll\left(\log _{2} x\right)^{-1 / 2+\delta / 2} .
$$

Proof. Let $\mathcal{E}(x)$ denote the set of integers less than or equal to $x$ with more than $\left(\log _{2} x\right)^{\delta / 2}$ distinct prime factors less than $y$ or more than $\left(\log _{2} x\right)^{\delta / 2}$ distinct prime factors greater than $z$. The size of this set is

$$
\begin{aligned}
|\mathcal{E}(x)| & \leq \frac{x}{\left\lfloor\left(\log _{2} x\right)^{\delta / 2}\right\rfloor !}\left(\sum_{p \leq y} \frac{1}{p}\right)^{\left(\log _{2} x\right)^{\delta / 2}}+\frac{x}{\left\lfloor\left(\log _{2} x\right)^{\delta / 2}\right\rfloor !}\left(\sum_{z \leq p \leq x} \frac{1}{p}\right)^{\left(\log _{2} x\right)^{\delta / 2}} \\
& \ll \frac{x}{\left(\log _{2} x\right)^{r}},
\end{aligned}
$$

using that $\sum_{p \leq x} \frac{1}{p}=\log _{2} x+C+O(1 / \log x)$ and Stirling's estimate $n ! \sim \sqrt{2 \pi} n^{n+\frac{1}{2}} e^{-n}$. For $n \notin \mathcal{E}(x)$ we have $\omega(n)-\omega(n ; y, z) \ll\left(\log _{2} x\right)^{\delta / 2}$, and so it follows that

$$
\begin{aligned}
& \frac{\omega(n)-\log _{2} x}{\sqrt{\log _{2} x}}-\frac{\omega(n ; y, z)-\sum_{y<p<z} \frac{1}{p}}{\sqrt{\sum_{y<p<z} \frac{1}{p}}} \\
& =\frac{\omega(n)-\log _{2} x-\omega(n ; y, z)+\sum_{y<p<z} \frac{1}{p}}{\sqrt{\sum_{y<p<z} \frac{1}{p}}}+O\left(\frac{\left|\omega(n)-\log _{2} x\right| \log _{3} x}{\log _{2} x}\right) \\
& \ll \frac{\left(\log _{2} x\right)^{\delta / 2}}{\sqrt{\log _{2} x}}+\frac{\left|\omega(n)-\log _{2} x\right| \log _{3} x}{\log _{2} x} .
\end{aligned}
$$

The proof is complete by noting that except for $O\left(x /\left(\log _{2} x\right)^{r}\right)$ integers less than $x$ we have $\left|\omega(n)-\log _{2} x\right| \ll\left(\log _{2} x\right)^{1 / 2+\delta / 4}$. This is because $\frac{\omega(n)-\log _{2} x}{\sqrt{\log _{2} x}}$ has normal moments and in particular $\frac{1}{x} \sum_{n \leq x}\left(\frac{\omega(n)-\log _{2} x}{\sqrt{\log _{2} x}}\right)^{8\lfloor r / \delta\rfloor} \ll 1$, where the implied constant depends on $r / \delta$. 


\section{Proof of Theorem 2.2}

Define for a prime $p$

$$
f_{p}(n)=\left\{\begin{array}{ll}
1-\frac{1}{p} & \text { if } p \mid n \\
-\frac{1}{p} & \text { if } p \nmid n
\end{array},\right.
$$

and if $m=\prod_{i} p_{i}^{\alpha_{i}}$, define $f_{m}(n)=\prod_{i} f_{p_{i}}(n)^{\alpha_{i}}$. (Thus $f_{1}(n)=1$.) If we think of a prime $p$ dividing $n$ with probability $1 / p$ independently of other primes, then we have $\mathbb{E}\left(f_{m}(n)\right)=0$ for square-free $m$. So we have written $\omega(n)-\sum_{p \leq x} \frac{1}{p}=\sum_{p \leq x} f_{p}(n)$ as a sum of independent random variables of mean 0 , which already suggests by the Central Limit Theorem that $\omega(n)-\sum_{p \leq x} \frac{1}{p}$ is normally distributed. This simple idea is actually very powerful. It is borrowed from Granville and Soundararajan [5], who use it to efficiently compute very high moments of $\omega(n)-\sum_{p \leq x} \frac{1}{p}$ and provide a new proof of the Erdös-Kac theorem.

We have that the left hand side of Theorem 2.2 equals

$$
\begin{aligned}
& \frac{1}{x} \sum_{n \leq x} \prod_{i=1}^{r} \exp \left(\mathbf{i} T_{i} \frac{\omega\left(n+b_{i} ; y, z\right)-\sum_{y<p<z} \frac{1}{p}}{\sqrt{\sum_{y<p<z} \frac{1}{p}}}\right) \\
& =\frac{1}{x} \sum_{n \leq x} \prod_{i=1}^{r} \exp \left(\sum_{y<p<z} \mathbf{i} t_{i} f_{p}\left(n+b_{i}\right)\right) \\
& =\frac{1}{x} \sum_{n \leq x} \prod_{y<p<z} \exp \left(\sum_{i=1}^{r} \mathbf{i} t_{i} f_{p}\left(n+b_{i}\right)\right) \\
& =\frac{1}{x} \sum_{n \leq x} \prod_{y<p<z}\left(1+\left(\sum_{i=1}^{r} \mathbf{i} t_{i} f_{p}\left(n+b_{i}\right)\right)+\frac{1}{2 !}\left(\sum_{i=1}^{r} \mathbf{i} t_{i} f_{p}\left(n+b_{i}\right)\right)^{2}+\ldots\right) .
\end{aligned}
$$

Now upon expansion of the product, (3.1) equals

$$
\frac{1}{x} \sum_{n \leq x} \sum_{\substack{a_{i} \geq 1 \\ p \mid a_{i} \Rightarrow y<p<z}} K_{a_{1}, \ldots, a_{r}} \prod_{i=1}^{r} t_{i}^{\Omega\left(a_{i}\right)} f_{a_{i}}\left(n+b_{i}\right),
$$

for some constants $K_{a_{1}, \ldots, a_{r}}$ of modulus bounded by 1 , where $\Omega(a)$ is the number of prime factors of $a$ counted with multiplicity. Note that when the integers $a_{i}$ are pairwise coprime we have that

$$
K_{a_{1}, \ldots, a_{r}}=\prod_{i=1}^{r} \prod_{p^{\alpha} \mid a_{i}} \frac{\mathbf{i}^{\alpha}}{\alpha !} .
$$

We will evaluate (3.2) using the following results. The first generalizes a result from [5].

Lemma 3.1. Let $a_{i}$ be pairwise coprime integers for $1 \leq i \leq r$. Denote the squarefree part of $a_{i}$ by $A_{i}$. We have

$$
\begin{gathered}
\frac{1}{x} \sum_{n \leq x} \prod_{i=1}^{r} f_{a_{i}}\left(n+b_{i}\right)=\prod_{i=1}^{r} \prod_{p^{\alpha} \| a_{i}}\left(\frac{1}{p}\left(1-\frac{1}{p}\right)^{\alpha}+\left(\frac{-1}{p}\right)^{\alpha}\left(1-\frac{1}{p}\right)\right) \\
+O\left(\frac{1}{x} \prod_{i=1}^{r} \tau\left(A_{i}\right)^{2}\right),
\end{gathered}
$$


where $\tau(A)$ denotes the number of divisors of $A$ and $p^{\alpha} \|$ a means that $p^{\alpha} \mid a$ and $p^{\alpha+1} \nmid a$. Note that the main term is zero unless each $a_{i}$ is square-full (that is, $p \mid a_{i}$ implies $\left.p^{2} \mid a_{i}\right)$.

Proof. For a fixed integer $a$ the value $f_{a}(n)$ depends only on the common prime factors of $a$ and $n$, so $f_{a}(n)=f_{a}((A, n))$. Thus we can group terms this way:

$$
\begin{aligned}
\frac{1}{x} \sum_{n \leq x} \prod_{i=1}^{r} f_{a_{i}}\left(n+b_{i}\right) & =\frac{1}{x} \sum_{d_{i} \mid A_{i}} \sum_{\substack{n \leq x \\
\left(A_{i}, n+b_{i}\right)=d_{i}}} \prod_{i=1}^{r} f_{a_{i}}\left(d_{i}\right) \\
& =\frac{1}{x} \sum_{d_{i} \mid A_{i}} \sum_{\substack{n \leq x \\
d_{i} \mid\left(n+b_{i}\right)}} \sum_{e_{i} \mid \frac{\left(A_{i}, n+b_{i}\right)}{d_{i}}} \prod_{i=1}^{r} \mu\left(e_{i}\right) f_{a_{i}}\left(d_{i}\right) \\
& =\frac{1}{x} \sum_{d_{i} \mid A_{i}} \sum_{e_{i} \mid \frac{A_{i}}{d_{i}}}\left(\sum_{\substack{n \leq x \\
e_{i} d_{i} \mid n+b_{i}}} 1\right) \prod_{i=1}^{r} \mu\left(e_{i}\right) f_{a_{i}}\left(d_{i}\right) .
\end{aligned}
$$

By the Chinese Remainder Theorem, since the integers $a_{i}$ are pairwise coprime, $\sum_{\substack{n \leq x \\ e_{i} d_{i} \mid n+b_{i}}} 1=\frac{x}{\prod_{i=1}^{r} e_{i} d_{i}}+O(1)$. Therefore the above sum is

$$
\begin{aligned}
& \prod_{i=1}^{r} \sum_{d_{i} \mid A_{i}} \frac{f_{a_{i}}\left(d_{i}\right)}{d_{i}} \sum_{e_{i} \mid A_{i}} \frac{\mu\left(e_{i}\right)}{d_{i}}+O\left(\frac{1}{x} \prod_{i=1}^{r} \tau\left(A_{i}\right)^{2}\right) \\
& =\prod_{i=1}^{r} \sum_{d_{i} \mid A_{i}} \frac{f_{a_{i}}\left(d_{i}\right)}{d_{i}} \frac{\phi\left(\frac{A_{i}}{d_{i}}\right)}{\frac{A_{i}}{d_{i}}}+O\left(\frac{1}{x} \prod_{i=1}^{r} \tau\left(A_{i}\right)^{2}\right) \\
& =\prod_{i=1}^{r} \sum_{d_{i} \mid A_{i}} f_{a_{i}}\left(d_{i}\right) \frac{1}{A_{i}} \phi\left(\frac{A_{i}}{d_{i}}\right)+O\left(\frac{1}{x} \prod_{i=1}^{r} \tau\left(A_{i}\right)^{2}\right) .
\end{aligned}
$$

Now it is easily verified (by multiplicativity in $a_{i}$ ) that the main term of the last line above equals

$$
\prod_{i=1}^{r} \prod_{p^{\alpha} \| a_{i}}\left(\frac{1}{p}\left(1-\frac{1}{p}\right)^{\alpha}+\left(\frac{-1}{p}\right)^{\alpha}\left(1-\frac{1}{p}\right)\right) .
$$

In the case that $a_{1}, \ldots, a_{r}$ are not pairwise coprime we will need the following.

Lemma 3.2. Let $r \geq 2$ and $0 \leq b_{1}<\ldots<b_{r} \leq \lambda\left(\log _{2} x\right)^{1 / 2-\delta}$. Suppose that for some prime $y<q<z$, we have that $q \mid a_{1}$ and $q \mid a_{2}$. Let $q^{\alpha_{i}} \| a_{i}$ and let $a_{i}^{\prime}=a_{i} q^{-\alpha_{i}}$. Let $A_{i}$ denote the squarefree part of $a_{i}$ and let $A_{i}^{\prime}$ denote the square free part of $a_{i}^{\prime}$. We have

$$
\frac{1}{x} \sum_{n \leq x} \prod_{i=1}^{r} f_{a_{i}}\left(n+b_{i}\right)=O\left(\frac{1}{q^{2}} \frac{1}{x} \sum_{n \leq x} \prod_{i=1}^{r} f_{a_{i}^{\prime}}\left(n+b_{i}\right)\right)+O\left(\frac{1}{q x} \prod_{i=1}^{r} \tau\left(A_{i}\right)^{2}\right) .
$$

Proof. From (3.4) we have

$$
\frac{1}{x} \sum_{n \leq x} \prod_{i=1}^{r} f_{a_{i}}\left(n+b_{i}\right)=\frac{1}{x} \sum_{d_{i} \mid A_{i}} \sum_{e_{i} \mid \frac{A_{i}}{d_{i}}}\left(\sum_{\substack{n \leq x \\ e_{i} d_{i} \mid n+b_{i}}} 1\right) \prod_{i=1}^{r} \mu\left(e_{i}\right) f_{a_{i}}\left(d_{i}\right) .
$$


The sum $\sum_{\substack{n \leq x \\ e_{i} d_{i} \mid n+b_{i}}} 1$ is zero for large enough $x$ if $q$ divides more than one integer $e_{i} d_{i}$. This is because if $q \mid n+b_{i}$ and $q \mid n+b_{j}$ then $q \mid b_{i}-b_{j}$ and hence $i=j$ since $q>y$ and the integers $b_{i}$ are distinct and bounded by $\lambda\left(\log _{2} x\right)^{1 / 2-\delta}$. Thus we can suppose that $q$ divides at most one integer $e_{i} d_{i}$. First consider the terms of (3.6) with $q \nmid e_{i} d_{i}$ for all $i$. These terms contribute

$$
\begin{aligned}
& \frac{1}{x} \sum_{d_{i} \mid A_{i}^{\prime}} \sum_{e_{i} \mid A_{i}^{\prime} / d_{i}}\left(\sum_{\substack{n \leq x \\
e_{i} d_{i} \mid n+b_{i}}} 1\right) \prod_{i=1}^{r} \mu\left(e_{i}\right) f_{a_{i}^{\prime}}\left(d_{i}\right) f_{q^{\alpha_{i}}}\left(d_{i}\right) \\
& =\left(\frac{-1}{q}\right)^{\alpha_{1}+\ldots+\alpha_{r}} \frac{1}{x} \sum_{n \leq x} \prod_{i=1}^{r} f_{a_{i}^{\prime}}\left(n+b_{i}\right),
\end{aligned}
$$

using that $f_{q^{\alpha_{i}}}\left(d_{i}\right)=(-1 / q)^{\alpha_{i}}$ and the identity of (3.6) . Since $\alpha_{1}+\alpha_{2} \geq 2$ we get the desired factor of $1 / q^{2}$. Now say $q \mid e_{1} d_{1}$ and $q \nmid e_{i} d_{i}$ for $i \neq 1$. The contribution of this case is,

$$
\begin{aligned}
& \frac{1}{x} \sum_{d_{i} \mid A_{i}^{\prime}} \sum_{e_{i} \mid A_{i}^{\prime} / d_{i}}\left(\sum_{\begin{array}{c}
n \leq x \\
q e_{1} d_{1} \mid n+b_{1} \\
e_{i} d_{i} \mid n+b_{i}, i \neq 1
\end{array}} 1\right) \mu\left(e_{1}\right) f_{a_{1}^{\prime}}\left(q d_{1}\right) f_{q^{\alpha_{1}}}\left(q d_{1}\right) \prod_{i=2}^{r} \mu\left(e_{i}\right) f_{a_{i}^{\prime}}\left(d_{i}\right) f_{q^{\alpha_{i}}}\left(d_{i}\right) \\
& +\frac{1}{x} \sum_{d_{i} \mid A_{i}^{\prime}} \sum_{e_{i} \mid A_{i}^{\prime} / d_{i}^{\prime}}\left(\sum_{\begin{array}{c}
n \leq x \\
q e_{1} d_{1} \mid n+b_{1} \\
e_{i} d_{i} \mid n+b_{i}, i \neq 1
\end{array}} 1\right) \mu\left(q e_{1}\right) f_{a_{1}^{\prime}}\left(d_{1}\right) f_{q^{\alpha_{1}}}\left(d_{1}\right) \prod_{i=2}^{r} \mu\left(e_{i}\right) f_{a_{i}^{\prime}}\left(d_{i}\right) f_{q^{\alpha_{i}}}\left(d_{i}\right),
\end{aligned}
$$

where the first line corresponds to $q \mid d_{1}$ and the second to $q \mid e_{1}$. We have by the Chinese Remainder Theorem,

$$
\sum_{\substack{n \leq x \\ q e_{1} d_{1}\left|n+b_{1} \\ e_{i} d_{i}\right| n+b_{i}, i \neq 1}} 1=\frac{1}{q} \sum_{\substack{n \leq x \\ e_{i} d_{i} \mid n+b_{i}}} 1+O(1) .
$$

Thus the contribution of the sums of (3.7) is

$$
\begin{aligned}
& \left(\frac{1}{q}\left(1-\frac{1}{q}\right)^{\alpha_{1}}\left(\frac{-1}{q}\right)^{\alpha_{2}+\ldots+\alpha_{r}}-\frac{1}{q}\left(\frac{-1}{q}\right)^{\alpha_{1}+\ldots+\alpha_{r}}\right) \frac{1}{x} \sum_{n \leq x} \prod_{i=1}^{r} f_{a_{i}^{\prime}}\left(n+b_{i}\right) \\
& \quad+O\left(\frac{1}{x}\left|f_{q^{\alpha_{2}}}\left(d_{2}\right)\right| \prod_{i=1}^{r} \sum_{e_{i} d_{i} \mid A_{i}^{\prime}} 1\right) .
\end{aligned}
$$

Again we have a factor of $1 / q^{2}$ in the first line above since $\alpha_{2} \geq 1$. The second line of (3.8) is $O\left(\frac{1}{q x} \prod_{i=1}^{r} \tau\left(A_{i}\right)^{2}\right)$ since $\alpha_{2} \geq 1$ and $q \nmid d_{2}$. This completes the proof as terms with $q \mid e_{j} d_{j}$ and $q \nmid e_{i} d_{i}$ for $i \neq j$ are dealt with similarly.

We will also need the following observations. 
Lemma 3.3. We have

$$
\frac{1}{x} \sum_{n \leq x} \sum_{\substack{a_{i} \geq 1 \\ p \mid a_{i}=y<p<z \\ \omega\left(a_{1}\right) \geq\left(\log _{2} x\right)^{2 r}}}\left|K_{a_{1}, \ldots, a_{r}}\right| \prod_{i=1}^{r}\left|f_{a_{i}}\left(n+b_{i}\right)\right|\left|t_{i}\right|^{\Omega\left(a_{i}\right)} \ll \frac{1}{\log x} .
$$

Proof. We first bound the contribution of terms with $\omega\left(a_{i}\right)=w_{i}$ for some positive integers $w_{i}$. Recall that $\left|K_{a_{1}, \ldots, a_{r}}\right| \leq 1$ and note that $\left|f_{p^{\alpha}}(n)\right| \leq\left|f_{p}(n)\right|$. Thus

$$
\begin{aligned}
& \frac{1}{x} \sum_{n \leq x} \sum_{\substack{a_{i} \geq 1 \\
p \mid a_{i} \Rightarrow y<p<z \\
\omega\left(a_{i}\right)=w_{i}}}\left|K_{a_{1}, \ldots, a_{r}}\right| \prod_{i=1}^{r}\left|f_{a_{i}}\left(n+b_{i}\right)\right|\left|t_{i}\right|^{\Omega\left(a_{i}\right)} \\
& \ll \frac{1}{x} \sum_{\substack { n \leq x \\
\begin{subarray}{c}{a_{i} \geq 1 \\
p \mid a_{i} \Rightarrow y<p<z \\
\omega\left(a_{i}\right)=w_{i}{ n \leq x \\
\begin{subarray} { c } { a _ { i } \geq 1 \\
p | a _ { i } \Rightarrow y < p < z \\
\omega ( a _ { i } ) = w _ { i } } }\end{subarray}} \prod_{i=1}^{r}\left|f_{A_{i}}\left(n+b_{i}\right)\right|\left|t_{i}\right|^{\Omega\left(a_{i}\right)}
\end{aligned}
$$

For a fixed square-free integer $A_{i}$ with $\omega\left(A_{i}\right)=w_{i}$ we have

$$
\sum_{\substack{a_{i} \geq 1 \\ A_{i}=\text { square-free part of } a_{i}}}\left|t_{i}\right|^{\Omega\left(a_{i}\right)} \leq 1,
$$

since $\left|t_{i}\right| \leq \frac{1}{1000}$. Thus (3.9) is bounded by

$$
\ll \frac{1}{x} \sum_{n \leq x} \prod_{i=1}^{r} \frac{1}{w_{i} !}\left(\sum_{y<p<z}\left|f_{p}\left(n+b_{i}\right)\right|\right)^{w_{i}} .
$$

Since $\left|f_{p}(n)\right| \leq 1$ if $p \mid n$ and $\left|f_{p}(n)\right| \leq \frac{1}{p}$ if $p \nmid n$, we have that (3.10) is bounded by

$$
\begin{aligned}
& \ll \frac{1}{x} \sum_{n \leq x} \prod_{i=1}^{r} \frac{1}{w_{i} !}\left(\omega\left(n+b_{i} ; y, z\right)+\log _{2} x\right)^{w_{i}} \\
& \ll\left(\prod_{i=1}^{r} \frac{1}{w_{i} !}\right) \frac{1}{x} \sum_{n \leq x} \sum_{i=1}^{r} 2^{r w_{i}}\left(\omega\left(n+b_{i} ; y, z\right)^{r w_{i}}+\left(\log _{2} x\right)^{r w_{i}}\right) .
\end{aligned}
$$

We have

$$
\frac{1}{x} \sum_{n \leq x} \omega\left(n+b_{i} ; y, z\right)^{r w_{i}} \ll \frac{1}{x} \sum_{y<p_{1}, \ldots, p_{r w_{i}}<z} \sum_{\substack{n \leq x \\\left[p_{1}, \ldots, p_{r w_{i}}\right] \mid n+b_{i}}} 1,
$$

where $\left[p_{1}, \ldots, p_{r w_{i}}\right]$ denotes the lowest common multiple of $p_{1}, \ldots, p_{r w_{i}}$. Now (3.12) is bounded by

$$
\ll \sum_{y<p_{1}, \ldots, p_{r w_{i}}<z} \frac{1}{\left[p_{1}, \ldots, p_{r w_{i}}\right]} \ll 2^{r w_{i}}\left(\log _{2} x\right)^{r w_{i}} .
$$

Thus we have that (3.11) is bounded by

$$
\ll\left(\prod_{i=1}^{r} \frac{1}{w_{i} !}\right) \sum_{i=1}^{r} 4^{r w_{i}}\left(\log _{2} x\right)^{r w_{i}} .
$$


Summing over integers $w_{i} \geq 1$ for $i \geq 2$ this is bounded by

$$
\ll \frac{\left(4 \log _{2} x\right)^{r w_{1}} \exp \left(\left(4 \log _{2} x\right)^{r}\right)}{w_{1} !} .
$$

Finally the sum of (3.15) over integers $w_{1} \geq\left(\log _{2} x\right)^{2 r}$ is $\ll 1 / \log x$.

Lemma 3.4. We have

$$
\sum_{\substack{a_{i} \geq 1 \\ p \mid a_{i} \Rightarrow y<p<z \\ \omega\left(a_{1}\right) \geq\left(\log _{2} x\right)^{2 r}}} \prod_{i=1}^{r}\left|t_{i}\right|^{\Omega\left(a_{i}\right)} \prod_{p^{\alpha} \| a_{i}}\left|\frac{1}{p}\left(1-\frac{1}{p}\right)^{\alpha}+\left(1-\frac{1}{p}\right)\left(\frac{-1}{p}\right)^{\alpha}\right| \ll \frac{1}{\log x} .
$$

Proof. We first bound the contribution of terms with $\omega\left(a_{i}\right)=w_{i}$ for some positive integers $w_{i}$. We have

$$
\begin{aligned}
& \sum_{\substack{a_{i} \geq 1 \\
p\left(a_{i} \Rightarrow y<p<z \\
\omega\left(a_{i}\right)=w_{i}\right.}} \prod_{i=1}^{r}\left|t_{i}\right|^{\Omega\left(a_{i}\right)} \prod_{p^{\alpha} \| a_{i}}\left|\frac{1}{p}\left(1-\frac{1}{p}\right)^{\alpha}+\left(1-\frac{1}{p}\right)\left(\frac{-1}{p}\right)^{\alpha}\right| \\
& \ll \prod_{i=1}^{r} \frac{1}{w_{i} !}\left(\sum_{y<p<z} \frac{1}{p}\right)^{w_{i}} \ll \prod_{i=1}^{r} \frac{\left(\log _{2} x\right)^{w_{i}}}{w_{i} !} .
\end{aligned}
$$

Summing over integers $w_{i} \geq 1$ for $i \geq 2$ this is bounded by

$$
\ll \frac{\left(\log _{2} x\right)^{w_{1}} \exp \left(r \log _{2} x\right)}{w_{1} !} .
$$

The sum of (3.15) over integers $w_{1} \geq\left(\log _{2} x\right)^{2 r}$ is $\ll 1 / \log x$.

Back to the proof. By Lemma 3.3 we see that (3.2) equals, up to an error of $O(1 / \log x)$,

$$
\frac{1}{x} \sum_{n \leq x} \sum_{\substack{a_{i} \geq 1 \\ p \mid a_{i} \Leftrightarrow y<p<z \\ \omega\left(a_{i}\right) \leq\left(\log _{2} x\right)^{2 r}}} K_{a_{1}, \ldots, a_{r}} \prod_{i=1}^{r} f_{a_{i}}\left(n+b_{i}\right) t_{i}^{\Omega\left(a_{i}\right)} .
$$

Let us first treat the terms of (3.18) with $a_{1}, \ldots, a_{r}$ not pairwise coprime. Applying Lemma 3.2 repeatedly, these terms contribute an amount bounded by

$$
\begin{gathered}
\ll \sum_{y<q<z} \frac{1}{q^{2}} \sum_{\substack{a_{i} \text { pairwise coprime } \\
p \mid a_{i} \Rightarrow y<p<z \\
\omega\left(a_{i}\right) \leq\left(\log _{2} x\right)^{2 r}}}\left|\frac{1}{x} \sum_{n \leq x} \prod_{i=1}^{r} f_{a_{i}}\left(n+b_{i}\right) t_{i}^{\Omega\left(a_{i}\right)}\right| \\
+\sum_{y<q<z} \frac{1}{q x} \sum_{\substack{a_{i} \geq 1 \\
p\left(a_{i} \Rightarrow y<p<z \\
\omega\left(a_{i}\right) \leq\left(\log _{2} x\right)^{2 r}\right.}} \prod_{i=1}^{r}\left|t_{i}\right|^{\Omega\left(a_{i}\right)} \tau\left(A_{i}\right)^{2} .
\end{gathered}
$$


For the second line of (3.19), we have

$$
\begin{aligned}
& \frac{1}{x} \prod_{i=1}^{r} \sum_{\substack{a_{i} \geq 1 \\
p \mid a_{i}=y<p<z \\
\omega\left(a_{i}\right) \leq\left(\log _{2} x\right)^{2 r}}}\left|t_{i}\right|^{\Omega\left(a_{i}\right)} \tau\left(A_{i}\right)^{2} \ll \frac{1}{x} \prod_{i=1}^{r} \sum_{\begin{array}{c}
a_{i} \text { square-free } \\
p \mid a_{i} \Rightarrow y<p<z \\
\omega\left(a_{i}\right) \leq\left(\log _{2} x\right)^{2 r}
\end{array}} \tau\left(A_{i}\right)^{2} \\
& \ll \frac{1}{x} \prod_{i=1}^{r} z^{\left(\log _{2} x\right)^{2 r}} 4^{\left(\log _{2} x\right)^{2 r}} \ll x^{-1 / 2} .
\end{aligned}
$$

Thus the second line of (3.19) falls into the error term of Theorem 2.2. To bound the first line of (3.19) we use Lemma 3.1 to get that

$$
\begin{aligned}
& \sum_{y<q<z} \frac{1}{q^{2}} \sum_{\substack{a_{i} \text { pairwise coprime } \\
p \mid a_{i} \Rightarrow y<p<z \\
\omega\left(a_{i}\right) \leq\left(\log _{2} x\right)^{2 r}}}\left|\frac{1}{x} \sum_{n \leq x} \prod_{i=1}^{r} f_{a_{i}}\left(n+b_{i}\right) t_{i}^{\Omega\left(a_{i}\right)}\right| \\
& \ll \frac{1}{y}\left(\sum_{\substack{a_{i} \geq 1 \\
p \mid a_{i} \Rightarrow y<p<z \\
\omega\left(a_{i}\right) \leq\left(\log _{2} x\right)^{2 r}}} \prod_{i=1}^{r}\left|t_{i}\right|^{\Omega\left(a_{i}\right)} \prod_{p^{\alpha} \| a_{i}} \frac{1}{p}+\sum_{\substack{a_{i} \geq 1 \\
p \mid a_{i} \Rightarrow y<p<z \\
\omega\left(a_{i}\right) \leq\left(\log _{2} x\right)^{2 r}}} \frac{1}{x} \prod_{i=1}^{r}\left|t_{i}\right|^{\Omega\left(a_{i}\right)} \tau\left(A_{i}\right)^{2}\right) \\
& \ll \frac{1}{y} \prod_{i=1}^{r} \prod_{y<p<z}\left(1+\frac{1}{p}\right)+x^{-1 / 2},
\end{aligned}
$$

where we used the bound of (3.20). Now this is less than $\frac{(\log x)^{r}}{y}+x^{-1 / 2} \ll 1 / \log x$.

Thus only the terms of (3.18) with $a_{1}, \ldots, a_{r}$ coprime will give a main contribution. Using Lemma 3.1 and (3.3) we get

$$
\begin{aligned}
& \frac{1}{x} \sum_{n \leq x} \sum_{\substack{a_{i} \\
p \text { pairwise coprime } \\
p\left(a_{i} \Rightarrow y<p<z \\
\omega\left(a_{i}\right) \leq\left(\log _{2} x\right)^{2 r}\right.}} K_{a_{1}, \ldots, a_{r}} \prod_{i=1}^{r} f_{a_{i}}\left(n+b_{i}\right) t_{i}^{\Omega\left(a_{i}\right)} \\
& =\prod_{\substack{i=1 \\
a_{i} \text { pairwise coprime } \\
p \mid a_{i} \Rightarrow y<p<z \\
\omega\left(a_{i}\right) \leq\left(\log _{2} x\right)^{2 r}}} \prod_{p^{\alpha} \| a_{i}} \frac{\mathbf{i}^{\alpha}}{\alpha !} t_{i}^{\alpha}\left(\frac{1}{p}\left(1-\frac{1}{p}\right)^{\alpha}+\left(1-\frac{1}{p}\right)\left(\frac{-1}{p}\right)^{\alpha}\right) \\
& +O\left(\sum_{\substack{a_{i} \geq 1 \\
p \mid a_{i} \Rightarrow y<p<z \\
\omega\left(a_{i}\right) \leq\left(\log _{2} x\right)^{2 r}}} \frac{1}{x} \prod_{i=1}^{r}\left|t_{i}\right|^{\Omega\left(a_{i}\right)} \tau\left(A_{i}\right)^{2}\right) .
\end{aligned}
$$

We've already seen in (3.20) that the error term above is negligible. The main term of (3.22) is zero if $a_{i}$ is not square-full for all $i$. Thus we may further impose the condition $p\left|a_{i} \Rightarrow p^{2}\right| a_{i}$. We may also extend by Lemma 3.4 the sum in the main term of (3.22) to all pairwise coprime and square-full integers $a_{i}$ whose prime factors lie between $y$ and $z$, up to an error of $O(1 / \log x)$. Thus (3.22) equals up to 
this error,

$$
\begin{aligned}
& \prod_{i=1}^{r} \prod_{y<p<z}\left(1+\frac{1}{p} \sum_{\alpha \geq 2} \frac{\mathbf{i}^{\alpha}}{\alpha !} t_{i}^{\alpha}\left(1-\frac{1}{p}\right)^{\alpha}+\left(1-\frac{1}{p}\right) \sum_{\alpha \geq 2} \frac{\mathbf{i}^{\alpha}}{\alpha !} t_{i}^{\alpha}\left(-\frac{1}{p}\right)^{\alpha}\right) \\
& =\prod_{i=1}^{r} \prod_{y<p<z}\left(1+\frac{1}{p}\left(e^{\mathbf{i} t_{i}}-1-\mathbf{i} t_{i}\right)+O\left(\frac{1}{p^{2}}\right)\right) \\
& =\prod_{i=1}^{r} \exp \left(\sum_{y<p<z} \log \left(1+\frac{1}{p}\left(e^{\mathbf{i} t_{i}}-1-\mathbf{i} t_{i}\right)+O\left(\frac{1}{p^{2}}\right)\right)\right) .
\end{aligned}
$$

Now since

$$
\log \left(1+\frac{1}{p}\left(e^{\mathbf{i} t_{i}}-1-\mathbf{i} t_{i}\right)+O\left(\frac{1}{p^{2}}\right)\right)=\frac{1}{p}\left(e^{\mathbf{i} t_{i}}-1-\mathbf{i} t_{i}\right)+O\left(\frac{1}{p^{2}}\right)
$$

we have that (3.23) equals

$$
\begin{aligned}
& \prod_{i=1}^{r} \exp \left(\left(e^{\mathbf{i} t_{i}}-1-\mathbf{i} t_{i}\right) \sum_{y<p<z} \frac{1}{p}+O\left(\frac{1}{y}\right)\right) \\
& =\prod_{i=1}^{r} \exp \left(\left(e^{\mathbf{i} t_{i}}-1-\mathbf{i} t_{i}\right) \sum_{y<p<z} \frac{1}{p}\right)+O(1 / \log x) .
\end{aligned}
$$

Acknowledgments. I am grateful to Prof. K. Soundararajan for posing the problem studied in this paper and for many helpful discussions pertaining to it. I am also thankful to Prof. A. Granville for numerous useful comments. Part of this work was done with support through a grant from the NSF (DMS 0500711) and while I visited the Centre de Recherches Mathématiques, Montréal.

\section{REFERENCES}

1. Cristian Cobeli and Alexandru Zaharescu, On the distribution of primitive roots mod $p$, Acta Arith. 83 (1998), no. 2, 143-153.

2. P. Erdös and M. Kac, The Gaussian law of errors in the theory of additive number theoretic functions, Amer. J. Math. 62 (1940), 738-742.

3. William Feller, An introduction to probability theory and its applications. Vol. II., Second edition, John Wiley \& Sons Inc., New York, 1971.

4. P. X. Gallagher, On the distribution of primes in short intervals, Mathematika 23 (1976), no. $1,4-9$.

5. A. Granville and K. Soundararajan, Sieving and the Erdös-Kac theorem, Proceedings of the NATO Advanced Study Institute on Equidistribution in Number Theory, Montréal, Canada, 11-22 July 2005, NATO Sci. Ser. II Math. Phys. Chem., vol. 237, Springer, pp. 15-27.

6. Andrew Granville and Pär Kurlberg, Poisson statistics via the Chinese remainder theorem, preprint.

7. Christopher Hooley, On the difference between consecutive numbers prime to n. II, Publ. Math. Debrecen 12 (1965), 39-49.

8. Rizwanur Khan, On the distribution of $\omega(n)$, Anatomy of integers, CRM Proc. Lecture Notes, vol. 46, Amer. Math. Soc., Providence, RI, 2008, pp. 199-207. MR MR2437977

9. Pär Kurlberg and Zeév Rudnick, The distribution of spacings between quadratic residues, Duke Math. J. 100 (1999), no. 2, 211-242.

10. A. Rényi and P. Turán, On a theorem of Erdös-Kac, Acta Arith. 4 (1958), 71-84.

11. Zeév Rudnick, Peter Sarnak, and Alexandru Zaharescu, The distribution of spacings between the fractional parts of $n^{2} \alpha$, Invent. Math. 145 (2001), no. 1, 37-57.

12. L. G. Sathe, On a problem of Hardy on the distribution of integers having a given number of prime factors. IV, J. Indian Math. Soc. (N.S.) 18 (1954), 43-81.

13. Atle Selberg, Note on a paper by L. G. Sathe, J. Indian Math. Soc. (N.S.) 18 (1954), 83-87. 
14. Gérald Tenenbaum, Introduction to analytic and probabilistic number theory, Cambridge Studies in Advanced Mathematics, vol. 46, Cambridge University Press, Cambridge, 1995, Translated from the second French edition (1995) by C. B. Thomas.

15. Pál Turán, On a theorem of Hardy and Ramanujan, J. London Math. Soc. 9 (1934), 274-276.

University of Michigan, Department of Mathematics, 530 Church St., Ann Arbor, MI 48109, USA

E-mail address: rrkhan@umich.edu 